\title{
Status of the KM3NeT project
}

\author{
Carla Distefano ${ }^{1, a}$ for the KM3NeT Collaboration \\ ${ }^{1}$ INFN Laboratori Nazionali del Sud, Via S.Sofia 62, 95123, Catania, Italy
}

\begin{abstract}
The KM3NeT Collaboration is constructing a research infrastructure hosting a next generation underwater neutrino observatory in the Mediterranean Sea. It will be a distributed infrastructure with two nodes: ORCA (Mton scale 2500 depth offshore Toulon (France)) and ARCA (Gton scale, $3500 \mathrm{~m}$ offshore Capo Passero (Italy)). The ORCA detector, optimised to detect neutrinos of tens of $\mathrm{GeV}$, aims at determining the neutrino mass hierarchy. ARCA is the high-energy component and is dedicated to the search for extraterrestrial neutrino sources in the TeV-PeV range. The KM3NeT Collaboration has started the construction of both detectors. The first detection units were deployed and the data analysis is ongoing to validate the detector performances. In this paper we give a general overview of the two detectors. We also report the present status of the detector and the first results of data analysis.
\end{abstract}

\section{Introduction}

KM3NeT is a research infrastructure hosting neutrino telescopes in the Mediterranean Sea. Two detectors with the same technology but different granularity are planned: ORCA (Oscillation Research with Cosmics in the Abyss) at a depth of $2500 \mathrm{~m}$ offshore Toulon, France and ARCA (Astroparticle Research with Cosmics in the Abyss) at a depth of $3500 \mathrm{~m}$ offshore Capo Passero, Italy.

The ORCA detector is optimised to measure neutrinos with energies at the GeV scale. Its primary goal is resolving a fundamental question of particle physics: the relative ordering of the neutrino masses, i.e. the question whether the third neutrino mass eigenstate is heavier (normal hierarchy, $\mathrm{NH}$ ) or lighter (inverted hierarchy, $\mathrm{IH}$ ) than the first and second states. The mass hierarchy influences the distortion of neutrino oscillation pattern due to the mass effects in the Earth. Looking at atmospheric neutrinos, ORCA has the possibility to measure the oscillation pattern distortion in a wide range of neutrino energies $(\mathrm{GeV}-\mathrm{PeV})$ and zenith angles, the latter corresponding to a wide range of baselines $(50-12800 \mathrm{~km})$.

The ARCA telescope is designed to observe high-energy neutrinos $(\mathrm{TeV} \div \mathrm{PeV})$ of cosmic origin. It will be the next generation of the underwater kilometre-cube scale neutrino telescopes. The main physics cases for ARCA are the search for diffuse neutrino fluxes, in particular the investigation from a complementary field of view of IceCube [1,2], and the search for point-like neutrino sources, with a particular focus on the galactic ones.

\footnotetext{
ae-mail: distefano_c@lns.infn.it
} 


\section{The KM3NeT detector design}

The KM3NeT detectors are three dimensional arrays of optical sensors detecting the Cherenkov light induced in water by the secondary charged particles produced in neutrino interactions.

The basic unit of the detectors is the Digital Optical Module (DOM) [3]. The DOM is a pressureresistant glass sphere of 17-inch diameter containing 31 3-inch photomultiplier tubes (PMTs) with their associated electronics for digitisation and data transmission as well as the calibration instrumentation. Nineteen of these PMTs are located in the lower hemisphere, and are thus looking downwards, while 12 in the upper hemisphere are looking upwards (Fig.1). This novel design offers significant improvements with respect to optical modules with a single large-area PMT: the total photocathode area is about three times larger, the field of view covers almost the full solid angle, pixelisation allows for high-purity photon counting and directional sensitivity.

A vertical sequence of 18 DOMs forms a Detection Unit (DU) [3]. Two thin parallel ropes hold the DOMs in place. The DU is anchored on the seabed and kept taut by a submerged buoy at its top. A vertical "backbone" cable, running along the full length of the DU, provides connectivity for power and data transmission. This cable is an oil-filled plastic tube, in equi-pressure with sea water, containing electrical wires and optical fibres, with a breakout box at each DOM. A sketch of the DU is shown in Fig.1.

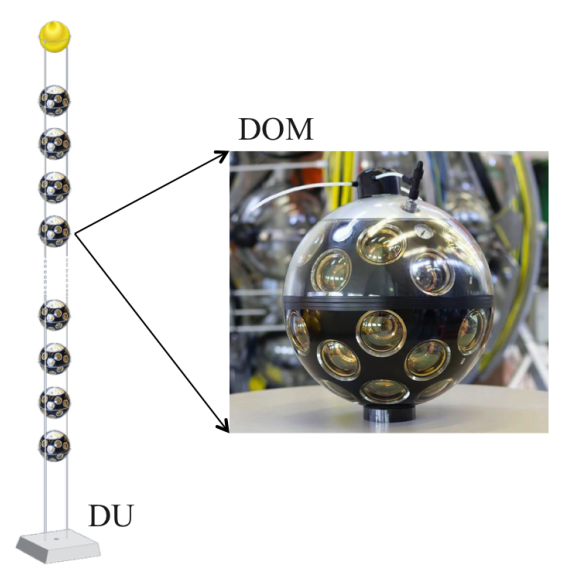

Figure 1. Schematic outline of a KM3NeT/ARCA building block.

A set of 115 DUs, arranged with a roughly circular footprint, forms a "building block". The DUs are connected to junction boxes and finally to shore by means of a main electro-optical cable. The architecture is intrinsically modular allowing for a staged implementation of the telescope. For the deployment the DU is coiled on a spherical aluminum frame with $2 \mathrm{~m}$ diameter, called Launcher of Optical Modules (LOM). In this compact configuration the DU is deployed on the seabed and connected to the seafloor network. The LOM is then released, unfurling the DU while rotating upwards. It floats to the surface where it is recovered for further use [3]. 


\section{The KM3NeT/ORCA detector}

The ORCA detector is located at the KM3NeT-France site off-shore Toulon and at a depth of $2450 \mathrm{~m}$ (see Fig. 2). The DOM spacing along the DU is $9 \mathrm{~m}$ and the average distance among the DUs is 23 $\mathrm{m}$. It will consists of one building block, as shown in Fig. 2, with an instrumented mass of 8 Mton.
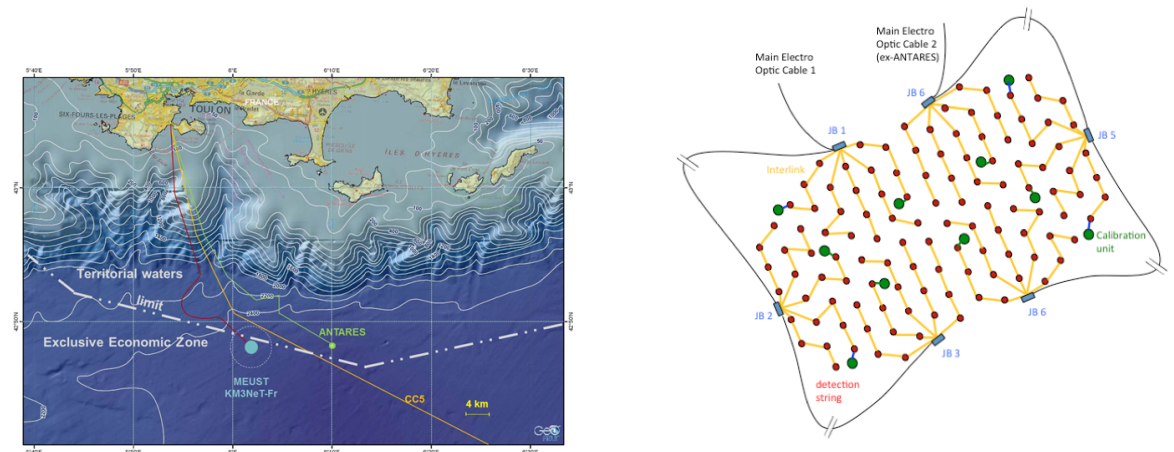

Figure 2. Left: map of the Mediterranean Sea south of Toulon, France. The location of the KM3NeT-France and ANTARES installations are indicated. Right: layout of the ORCA detector, depicting the 115 DUs, cables and connection devices of the full array.

The arrival times of the light collected by optical sensors and their positions are used to reconstruct the secondary particles. From these the neutrino arrival directions can be inferred and their energies estimated. An angular resolution of $5^{\circ}$ and and energy resolution of $30 \%$ at $10 \mathrm{GeV}$, for both track and shower channels, are estimated for ORCA with Monte Carlo simulations. An algorithm based on the Random Decision Forest technique is used to perform particle identification. Monte Carlo simulations show that $90 \%$ of electron neutrinos and $70 \%$ of muon neutrinos are correctly identified at $10 \mathrm{GeV}$ (Fig. 3). The algorithm is also able to suppress atmospheric muon background and noise to $3 \%$ level, preserving $95 \%$ of the neutrino signal. With a LLR test statistic is then possible to estimate the expected sensitivity to exclude the wrong hierarchy. The ORCA detector could be able to resolve the question of the neutrino mass ordering after only 3 years of data taking, as shown in Fig. 3.

\section{The KM3NeT/ARCA detector}

The ARCA telescope is located at the KM3NeT-Italy site, about $100 \mathrm{~km}$ off-shore Portopalo di Capo Passero (Sicily), at a depth of $3500 \mathrm{~m}$ (see Fig. 4). The DOM spacing along the DU is $36 \mathrm{~m}$ with an average distance among DUs of $90 \mathrm{~m}$. ARCA will consists of two building blocks as shown in Fig. 4 and will have an instrumented volume of $1 \mathrm{~km}^{3}$.

The golden channel is the detection of long track muon produced in CC muon neutrino interaction. For this kind of events an angular resolution better than $0.1^{\circ}$ and an energy resolution of $30 \%$ in $\log$ energy are reached for neutrino energies greater than $10 \mathrm{TeV}$. Shower events, induced by all flavour neutrino interaction in $\mathrm{NC}$ and electron neutrinos in $\mathrm{CC}$, are also detectable. Monte Carlo simulations show that showers events will be reconstructed with an angular resolution better than $2^{\circ}$ and energy resolution better $\approx 5 \%$ at $1 \sigma$.

The identification of point-like sources is one of the main goals of the ARCA telescope, for which the sensitivity to a generic $E^{-2}$ neutrino flux is shown in Fig. 5. Thanks to its geographical location, 

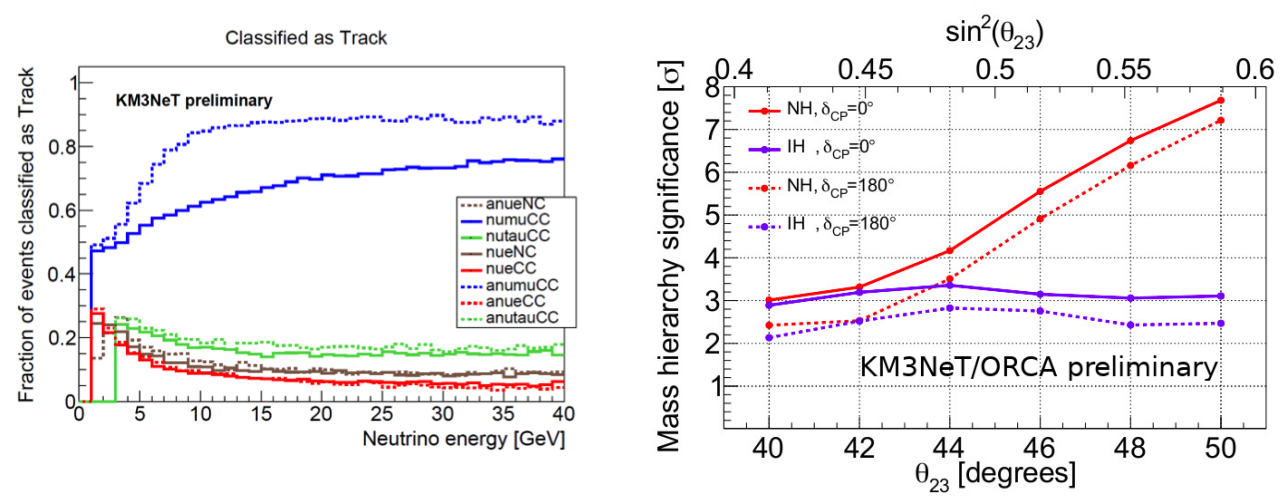

Figure 3. Fraction of events classified as tracks with the ORCA detector (left) and detector sensitivity to reject the wrong hierarchy after 3 years of data taking (right).
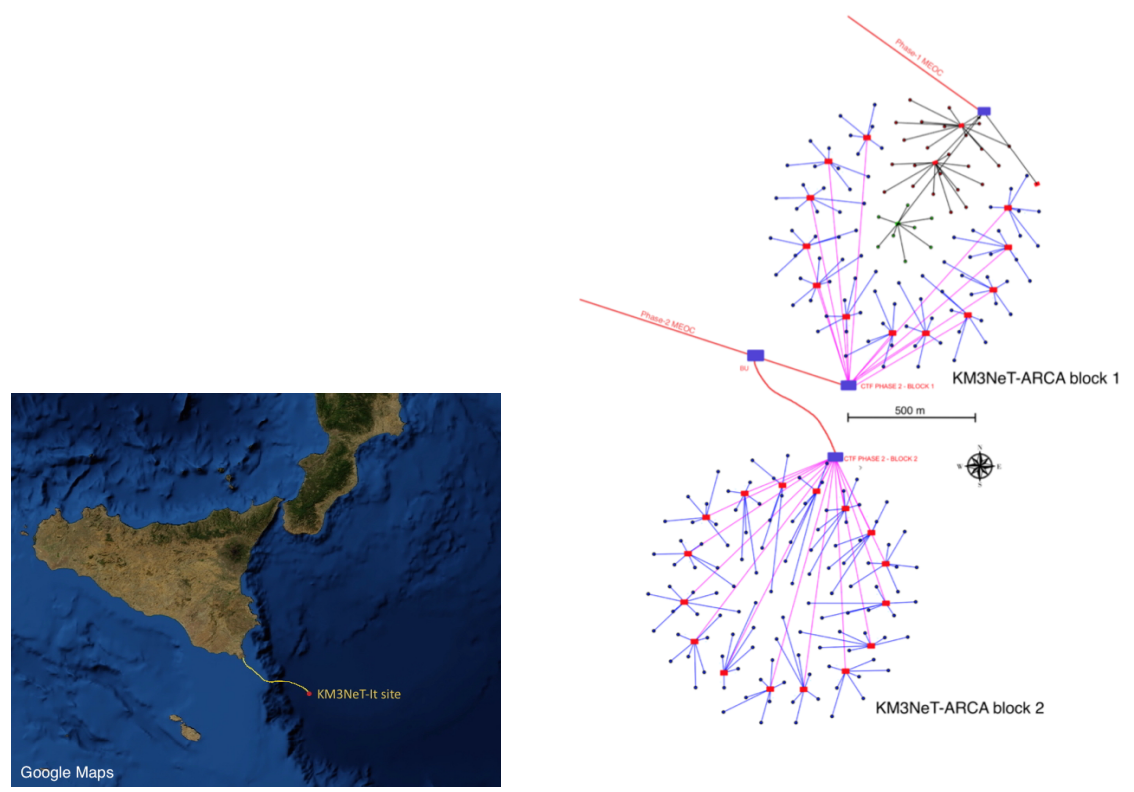

Figure 4. Left: map of the Mediterranean Sea south of Sicily, Italy. The location of the KM3NeT-Italy is indicated. Right: layout of the ARCA detector, depicting the two building blocks, cables and connection devices.

the telescope will allow for surveying a large part of the Galactic Plane, including the Galactic Centre. The sensitivity for specific galactic sources are shown in the right panel of Fig. 5.

\section{First results}

The KM3NeT detectors are currently under construction. The first two strings of ARCA were deployed in December 2015 and in May 2016 and the first string of ORCA was deployed in September 

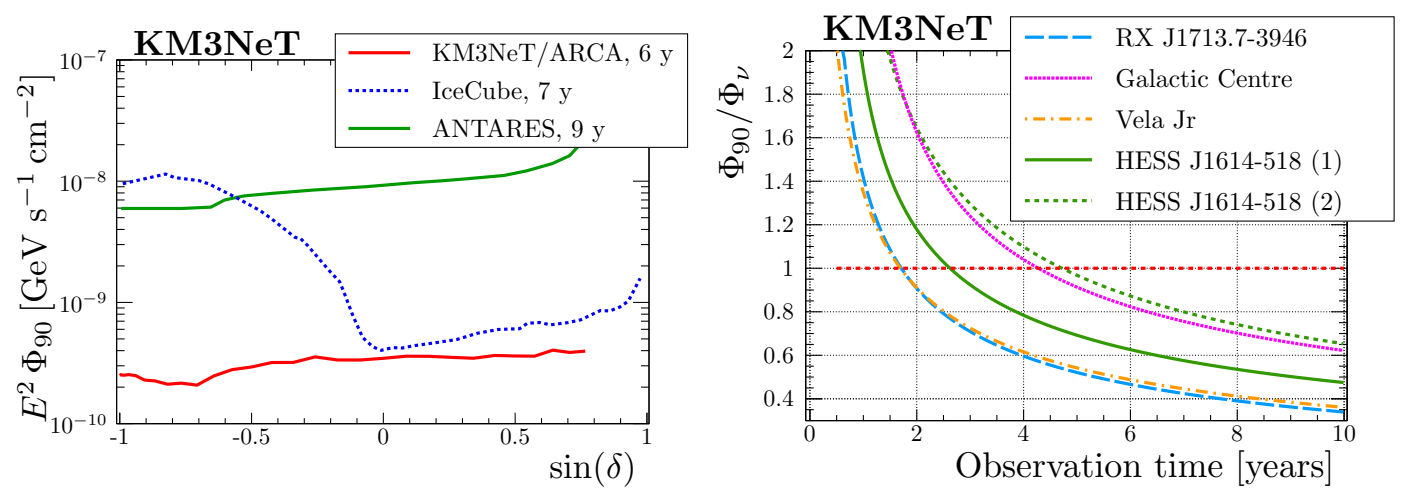

Figure 5. Left: ARCA sensitivity for a generic, unbroken $E^{-2}$ neutrino flux as a function of the source declination. For comparison, the corresponding IceCube [4] and ANTARES [5] results are also shown. Right: ratio of ARCA sensitivity to the expectation flux as a function of the observation time for some galactic sources. See ref. [6] for details.

2017. Data collected since their deployment were analysed to perform in-situ calibrations to determine the relative PMT efficiencies and PMT-specific transit times ('intra-DOM calibration') and to measure the DOM specific optical fibre lengths ('inter-DOM calibration') [7].

A measurement of the depth dependence of the coincidence rates induced by atmospheric muons looking at the coincidence multiplicity occurring in the different DOMs is on going [8]. Results for the two ARCA DUs are shown in Fig.6. The good agreement between the data and the Monte Carlo simulations confirms that the measured coincidence rate as a function of the depth matches the expected behaviour due to the depth dependence of the atmospheric muon flux.
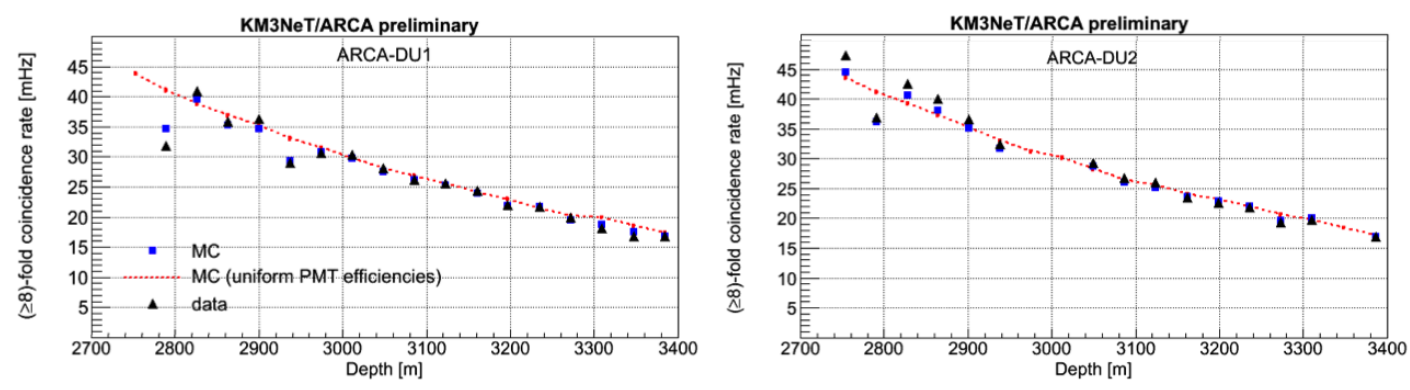

Figure 6. $\geq 8$-fold coincidence rate as a function of the depth for the two ARCA DUs. The black points are the data. The red dashed lines are the base expectation from an ideal Monte Carlo with uniform PMT efficiencies which neglects the differences between individual PMTs as determined from in-situ calibration. The blue points are the Monte Carlo simulations where PMT efficiencies have been taken into account.

Data collected are also under analysis to reconstruct atmospheric muons and neutrinos in order to check the reconstruction algorithms and to optimise the event selection criteria for atmospheric muon rejection. The angular distribution of the events reconstructed with the 82 days live time data acquired with the ORCA DU are shown in Fig. 7. 


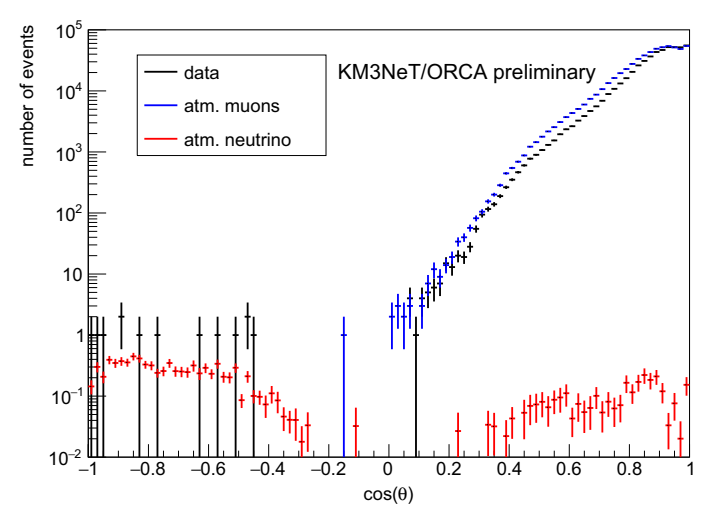

Figure 7. Angular distribution of events detected by the ORCA DU in a live time of 82 days, compared with Monte Carlo simulations.

\section{Conclusions}

The construction of the KM3NeT detectors has begun in the Mediterranean sea. The ORCA detector, devoted to study the atmospheric neutrino oscillations, will be able to determine the neutrino mass hierarchy in a few years of data taking. The ARCA telescope, with an instrumented volume of $1 \mathrm{~km}^{3}$, will offer independent confirmation of the IceCube flux and it will be optimised to study point-like astrophysical neutrino sources. In particular, it will profit of the advantage of its location to observe the Galactic Plane and the Galactic Centre.

The first two ARCA DUs and the first ORCA DU were deployed between 2015 and 2017 and data collected since their deployment are under analysis. A measurement of the depth dependence of the PMT coincidence rates induced by atmospheric muons is on-going. Data are also under analysis to reconstruct atmospheric muons and neutrinos in order to check the reconstruction algorithms and to optimise the event selection criteria for atmospheric muon rejection. First results show a good agreement with Monte Carlo simulations and then the functionality of the detector.

\section{References}

[1] M.G. Aartsen et al. (IceCube), Science 342, 1242856 (2013)

[2] M.G. Aartsen et al. (IceCube), Science 361, 147 (2018)

[3] S. Adrián-Martínez et al. (KM3NeT), J. Phys. G: Nucl. Part. Phys 43, 084001 (2016)

[4] M.G. Aartsen et al. (IceCube), Astroph. J. 835, 151 (2017)

[5] A. Albert et al. (ANTARES), Phys. Rev. D 96, 082001 (2017)

[6] S. Aiello et al. (KM3NeT), submitted to Astrop. Phys. (2018), arXiv: 1810.08499

[7] K. Melis (KM3NeT), Proc. of Science, PoS(ICRC2017)1059 (2017)

[8] M. Jongen (KM3NeT), Proc. of Science, PoS(ICRC2017)1018 (2017) 